\title{
Novel Multiple Impression based Multimodal Fingerprint Recognition System
}

\author{
Arjun V Mane \\ Department of Computer \\ Science \& IT, Dr.Babasaheb \\ Ambedkar Marathwada \\ University, Aurangabad (MS), \\ India
}

\author{
Yogesh S Rode \\ Department of Computer \\ Science \& IT, Dr.Babasaheb \\ Ambedkar \\ Marathwada University, \\ Aurangabad (MS), India
}

\author{
K V Kale \\ Department of Computer \\ Science \&IT, Dr. Babasaheb \\ Ambedkar \\ Marathwada University, \\ Aurangabad (MS), India
}

\begin{abstract}
As population grow, the task of fingerprint verification system becomes increasingly more difficult. However performance improvement of biometric based verification system is an interesting and emerging area. In fingerprint verification systems, there are usually multiple enrolled impressions for a same finger. The performance of the systems can be improved by combining these impressions at enrollment level as well as testing level. In this research paper we use a method of score level fusion using multiple enrollment and multiple testing impressions to achieve higher accuracy. Experimental results show that a larger improvement can be obtained by using fusion of multiple enrollments and one testing impression scheme than other one to one matching. Experiment conducted with FVC2000, FVC 2002 and our own (BAMU) database. For the evaluation performance measures (FAR), (FRR), Recognition rate (RR) Equal Error Rate (EER) and Receiver Operating Characteristics curve (ROC) has been used.
\end{abstract}

\section{Keywords}

Fingerprint Recognition, Multiple impressions, Fusion.

\section{INTRODUCTION}

We Many researchers have shown that the performance using a single fingerprint, a single feature or a single classifier usually cannot meet the need of most practical application, and much work has been done by using multimodal biometric [1]. Fingerprint based authentication system is reliable in large scale identification applications such as law enforcement, border control, background check [2]. Fingerprint based recognition is the most popular method in biometric community [3]. There is no two fingerprints are never identical in every details, even two fingerprints are recorded immediately after each other [4].We used multiple impressions as a multiple biometric traits. Under this research we combine matching score of multiple instance of same finger collected by same fingerprint sensor, because use of two different sensors and different biometric traits can increase system verification time and inconvenience to the user. This research paper is intended to show the difference between results of two biometric systems one based on single finger print impression matching and second one is based on multiple

\section{RELATED WORK}

Combination of multiple impressions of same finger has been proposed in several researchers: Prabhakar and Jain
(2002) [5] combine multiple impression of a finger for verification system and show the good improvement in recognition accuracy. This idea was further developed by Simon-Zorita et al. (2003) who proposed to store three impressions of finger during enrollment, during verification, the image is compared with all the three enrollment impressions and the maximum score is considered the fusion score. L. Sha ref [6] used multiple impressions of same finger to consolidate the outcome of fingerprint matching; they have implemented matching at two level one at matching score level and second at decision level. Chybxiao and yilong ref [7] they used novel method of score level fusion using multiple impressions for fingerprint verification.

\section{FINGERPRIENT}

A fingerprint is the pattern of ridges and valleys on the surface of the finger [8]. Fingerprint recognition is one of the most popular and successful methods used for person identification. Biometric identification systems using fingerprints patterns, is called automatic fingerprint identification system.

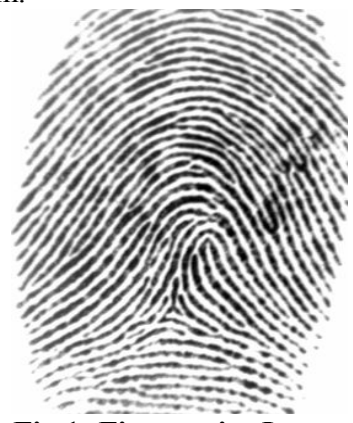

Fig 1: Fingerprint Image

The three basic patterns of fingerprint ridges are the arch, loop, and whorl [9]. This basic pattern or texture information can be use as local or global features in fingerprint recognition.

\section{EXPERIMENTS}

Many research works has been done using multiple features, multiple matchers, multiple fingers and multiple impressions of a same finger. In this paper, more information is utilized by using score level fusion of multiple impressions at enrollment stage as well as testing stage. This paper is address on comparative study of single one to one fingerprint impression matching and by combining multiple impressions of a fingerprint at 
enrollment and testing level. For feature extraction and matching we follow the Filter-Bank Base Fingerprint Matching Algorithm which is upholding in ref. [4].This Algorithm go through following steps.
a. Read the fingerprint image.
b. Image Enhancement
c. Reference Point location
d. Tessellate Region of Interest
e. Filter the region of interest in eight different directions using a bank of Gabor filters
f. Compute the average absolute deviation from the mean $(\mathrm{AAD})$ of gray values to define the feature vector or the FingerCode.
g. Matching
h. Calculate Matching Score

First determine a reference point and region of interest for the read fingerprint image. The reference point is taken to be the center point in a fingerprint which is defined as the point of maximum curvature of the ridges in a fingerprint. The region of interest is a circular area around the reference point. Tessellate the region of interest. The region of interest is divided into sectors and the gray values in each sector are normalized to a constant mean and variance. Filter the region of interest in eight different directions using a bank of Gabor filter. Gabor filter has the following form in the spatial domain.

$$
\begin{aligned}
G(x, y, f, \theta)= & \exp \left\{\frac{-1}{2}\left[\frac{x^{\prime 2}}{\delta_{x}{ }^{2}}+\frac{y^{2}}{\delta_{y}{ }^{2}}\right]\right\} \cos \left(2 \pi f x^{\prime}\right) \\
x^{\prime} & =x \sin \theta+y \cos \theta, \\
y & =x \cos \theta+y \sin \theta
\end{aligned}
$$

Filtering produces a set of eight filtered images. Compute the average absolute deviation from the mean (AAD) of gray values in individual sectors in each filtered image. $\mathrm{AAD}$ value in each sector quantifies the underlying ridge structures and is defined as a feature. A feature vector, which we call FingerCode, is the collection of all the features in each filtered image. Thus, the feature elements capture the local information and the ordered enumeration of the tessellation captures the invariant global relationships among the local patterns. The representation is invariant to translation of the image. It is assumed that the fingerprint is captured in an upright position and the rotation invariance is achieved by storing 10 representations corresponding to the various rotations $(-45: 00 ;-450 ;-33: 750 ;-22: 50 ;-11: 250$; $00 ; 11: 250,22: 50 ; 33: 750 ; 45: 00)$ of the image. This algorithm more clear in ref. [4].

\subsection{Score-Level Fusion}

Score-level fusion: In score-level fusion the match scores output by multiple biometric matchers are combined to generate a new match score (a scalar) that can be subsequently used by the verification or identification modules for rendering an identity decision The match score is a measure of similarity between the input and template feature vectors. This is also known as fusion at the measurement level or confidence level [10] [11]. Fusion at this level is the most commonly discussed approach in the biometric literature primarily due to the ease of accessing and processing match scores. There are Sum of scores, Maxscore, Min-score techniques for score level fusion.

\subsection{Matching Algorithm}

The score produced after matching is a real value between 0 and 1, which represents the similarity degree of two sets. The maximum value indicates that the compared fingerprint impression belong to the same finger, the minimum value indicates that the fingerprints impressions are different. Enrollment and Testing Stage: we use multiple impressions of same finger for Enrollment as well as testing stage as:

$$
E=\left\{I^{i} \mid i=1,2,3 \ldots n\right\}
$$

Where $\mathbf{I}^{\mathbf{i}}$ is $\mathbf{i}^{\text {th }}$ impression and $\mathbf{n}$ represent $\mathbf{n}$ number of impressions for enrollment or testing. For combining two enrolled impressions that is Similarity $\left(\mathbf{I}^{\mathbf{i}}, \mathbf{I}^{\mathbf{j}}\right)$ multiple impressions we calculate similarity between by matching with each other. Set of similarity is represented as;

$$
\begin{gathered}
S_{\text {sim }}=\left\{\text { Similarity }\left(\left(I^{i}, I^{j}\right)\right) \mid I^{i}, I^{j} \in E\right\} \\
i=1,2, \ldots . n, \\
j=1,2, \ldots . . m,
\end{gathered}
$$

Where Ssim having $\mathbf{N}$ number of similarity. Let $\mathbf{X}$ be the template feature set. Let $\mathbf{Y}$ be the input feature set. For each feature value $\mathbf{x} \in \mathbf{X}$, the following algorithm is performed. For each $\mathbf{y} \in \mathbf{Y}$, After this $\mathrm{x}$ match with $\mathrm{y}$. At the end of these loops, the value $\max \{|\boldsymbol{A}(\boldsymbol{x}, \boldsymbol{y})|\}$ is converted to the matching score by the formula

$$
\text { Score }=\frac{\max \{|A(x, y)|\}}{|X| .|Y|}
$$

Apply the following transformation to the above scores $\boldsymbol{S}_{\boldsymbol{0}}$ and $\boldsymbol{S}_{\boldsymbol{c}}$ to implement the fusion:

$$
S=\operatorname{fusion}\left(S_{0}, S_{c}\right)
$$

Compare the obtained score value $\mathrm{S}$ with a threshold.

$$
S>\text { threshold }
$$

If the value of $\mathrm{S}$ is larger than a given threshold, the claimant is accepted; otherwise, it will be rejected. Our experiment based on four different categories for enrollment and testing, i.e., One enrollment One testing, One Enrollment and Multiple Testing, Multiple Enrollment and One Testing, Multiple Enrollment and Multiple Testing

i. One Enrollment One Testing: This is unimodal biometric system further we compare these results with proposed multimodal biometric system. In this system we use one fingerprint impression features for enrollment and one fingerprint impression for matching, hence matching score obtain, compare this with threshold. This is one to one matching show in following figure. 


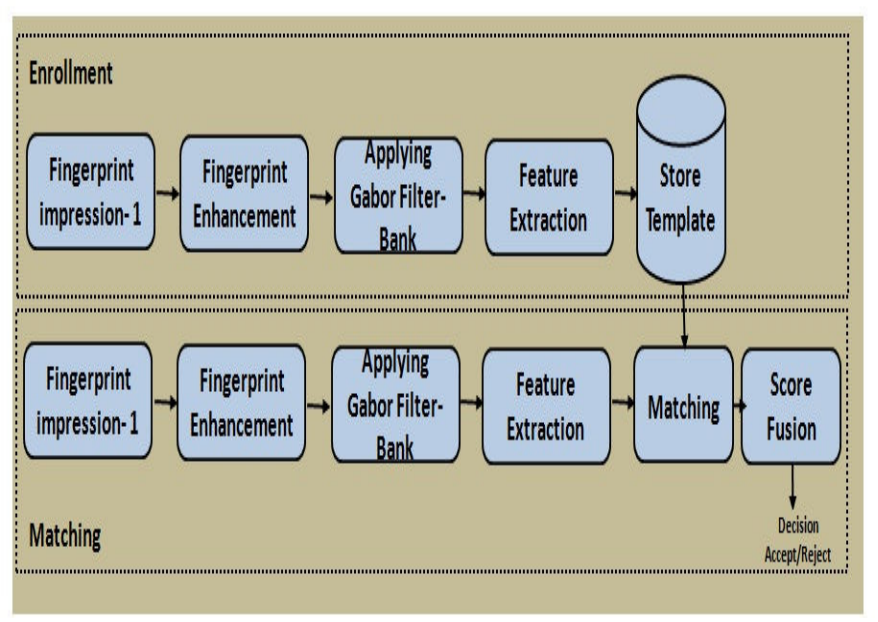

Fig 2: One enrollment One testing

ii. One Enrollment Multiple Testing: This is the next step where we use one fingerprint impression for enrollment and combination of multiple impressions for testing. Here we use multiple impressions at testing stage which is similarity two impressions by matching with each other. We match this similarity with single enrolled impression and matching score is calculated and decision is taken. Following figure shows this

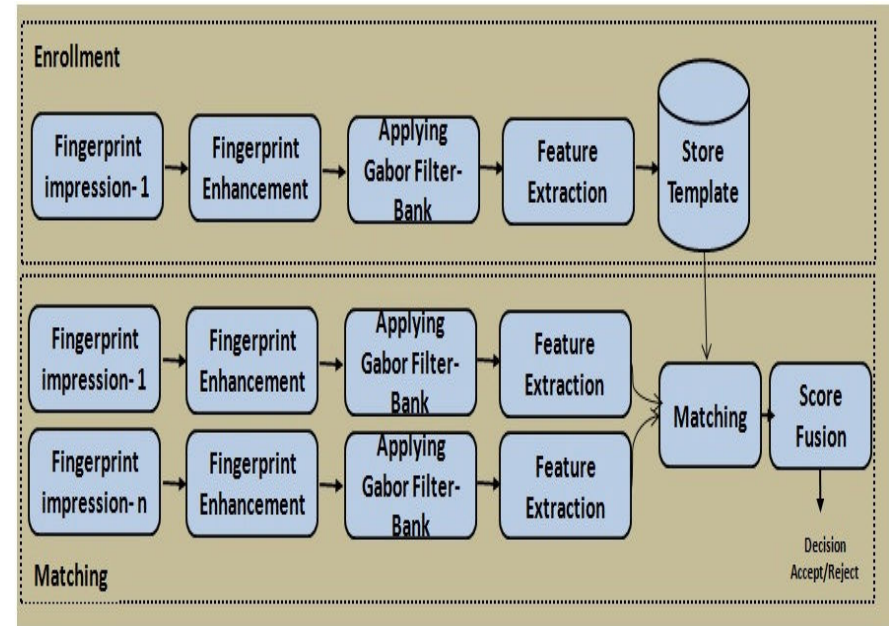

Fig 3: One enrollment Multiple testing

iii. Multiple Enrollment One Testing: Same way here in this stage we use similarity of two fingerprint impression after matching as a template and one impression for testing and calculated matching score, this multiple enrollment and one testing and decision is taken by compare the obtained score value with a threshold. Following figure how this matching.

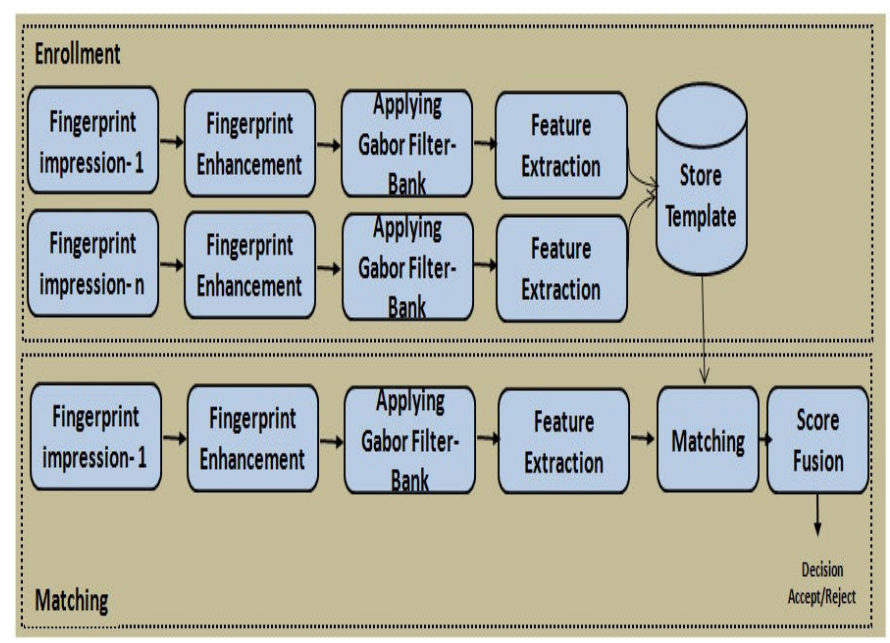

Fig 4: Multiple enrollment One testing

iv. Multiple enrollment Multiple testing: At the end we use similarity of two impression after matching as a template and similarity of two impression after matching as a testing and obtain score is compare with threshold and decision taken following figure shows same.

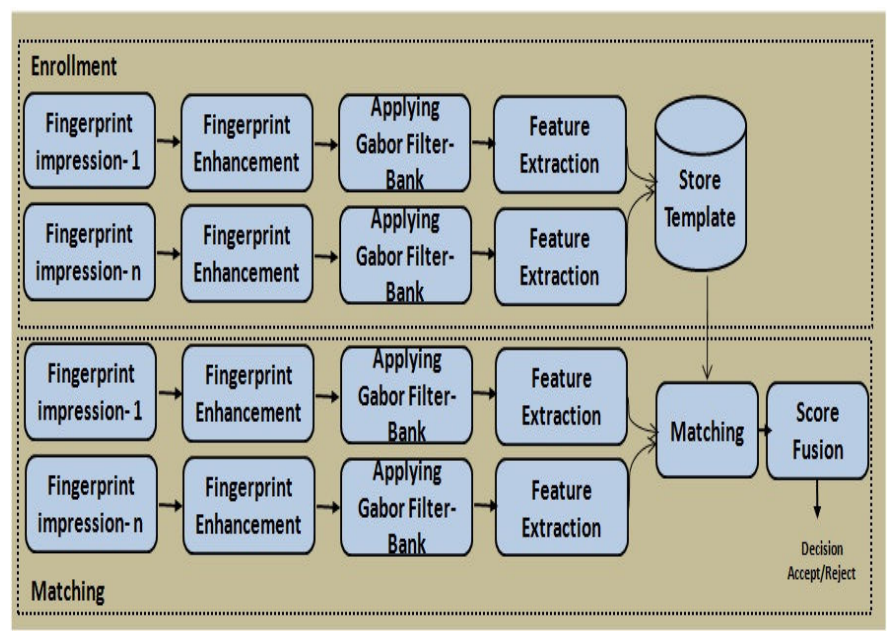

\section{RESULTS}

Four databases are used for our experiments, including our own BAMU database and three standard databases, FVC'2000 DB1 [12]. FVC'2002 DB1 [13]. FVC'2004 DB1 [14].BAMU database having 660x4 images, (images size $=248 \times 292,500$ dpi) capture using is an optical USB fingerprint scanner. These fingerprint images vary in different way like oily, dry, different rotation. FVC'2000 DB1, FVC'2002 DB1, FVC'2004 DB1 having 100x8 images and all description about these databases are given in corresponding references. We enhance all these database images using algorithm in ref. [15]. In the experiment, we will mainly compare the performance of fusion of multiple impressions at different level. We have evaluated our results by calculating False Acceptance Rate (FAR), False Rejection Rate (FRR), Equal Error Rate (EER), Recognition rate (RR) and Receiver Operating Characteristics curve (ROC). 


\begin{tabular}{|c|c|c|c|c|c|c|c|c|}
\hline \multirow{2}{*}{ Algorithm } & \multicolumn{2}{|c|}{ FVC2000_DB1 } & \multicolumn{2}{c|}{ FVC2002_DB1 } & \multicolumn{2}{c|}{ FVC2004_DB1 } & \multicolumn{2}{c|}{ BAMU_DB } \\
\cline { 2 - 9 } & RR & EER & RR & EER & RR & EER & RR & EER \\
\hline Filterbank 1-1 & 75.00 & 17.50 & 80.00 & 17.00 & 38.00 & 57.00 & 89.50 & 07.00 \\
\hline Filterbank 1-n & 76.50 & 8.33 & 80.00 & 15.50 & 38.00 & 57.00 & 89.50 & 07.00 \\
\hline Filterbank n-1 & 83.00 & 6.00 & 88.00 & 10.00 & 39.00 & 51.00 & 99.00 & 00.00 \\
\hline Filterbank n-n & 80.50 & 13.7 & 80.00 & 12.00 & 46.50 & 44.50 & 93.00 & 03.00 \\
\hline
\end{tabular}

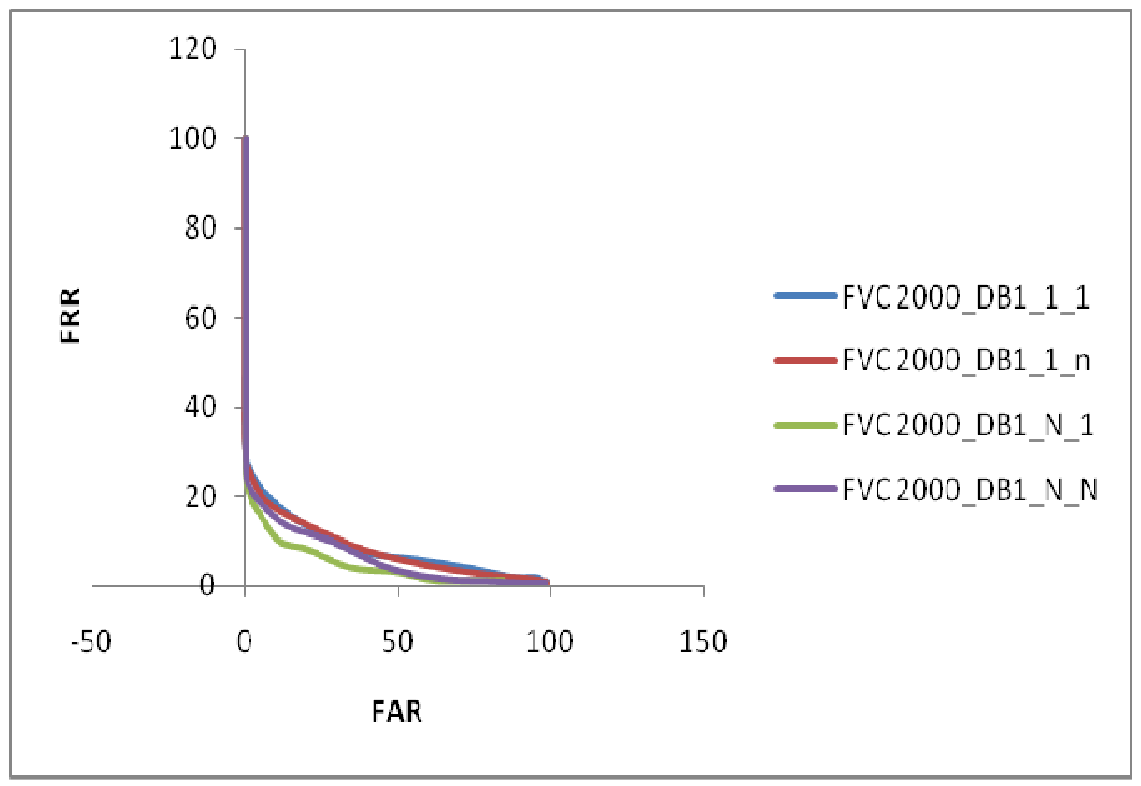

Fig.6: ROC FVC 2000_DB1

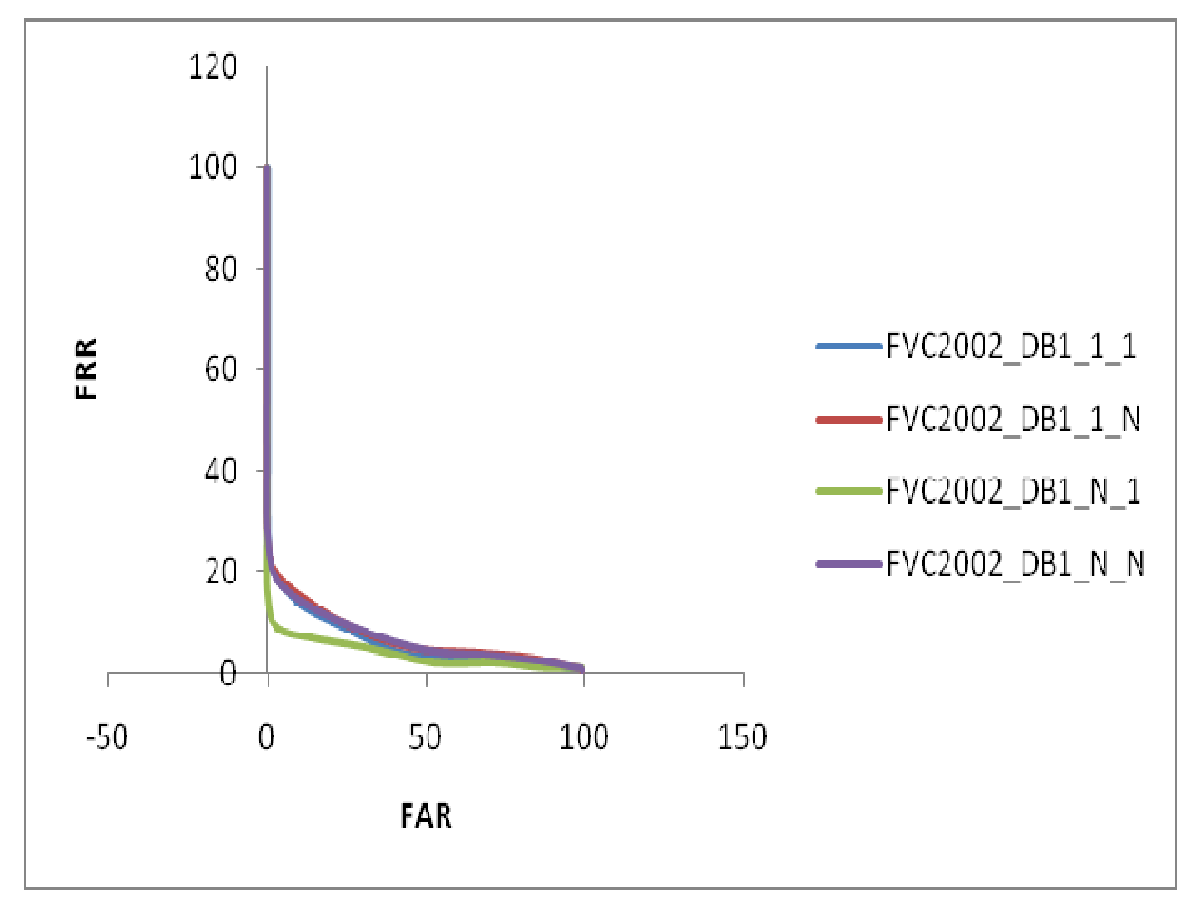

Figure7: ROC of FVC_2002_DB1 


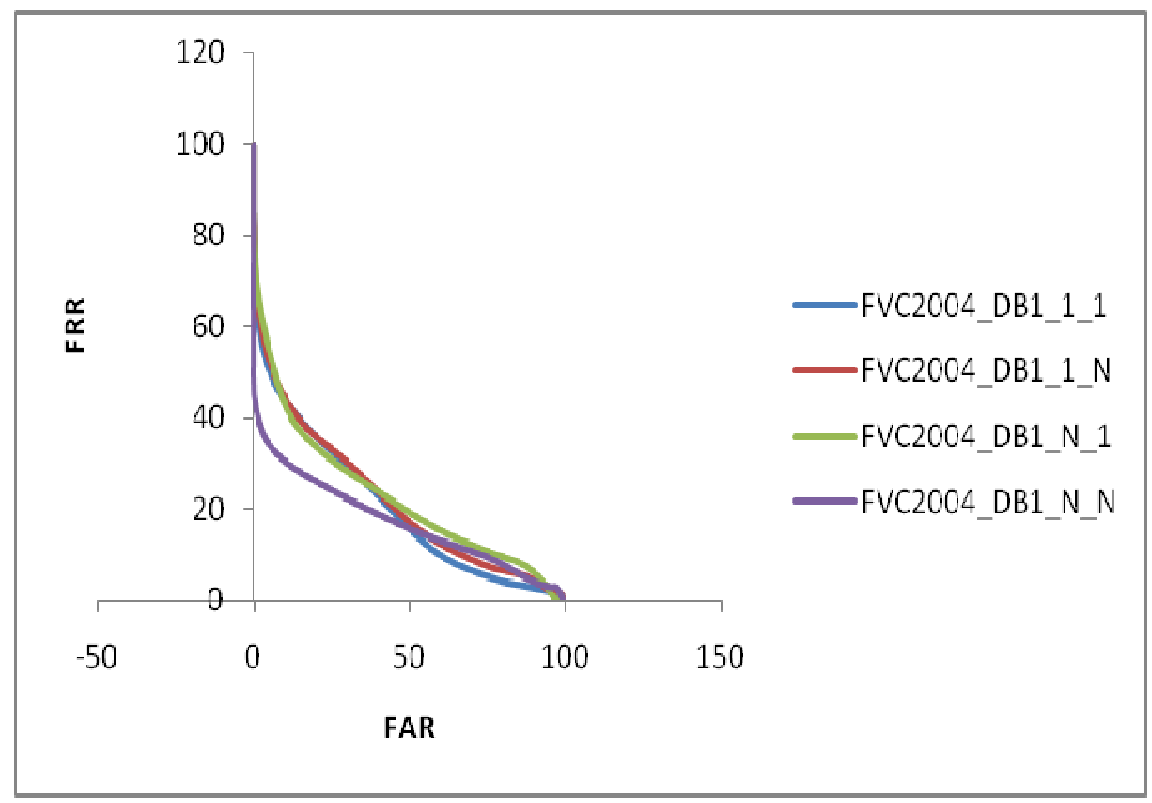

Figure8: ROC of FVC_2004_DB1

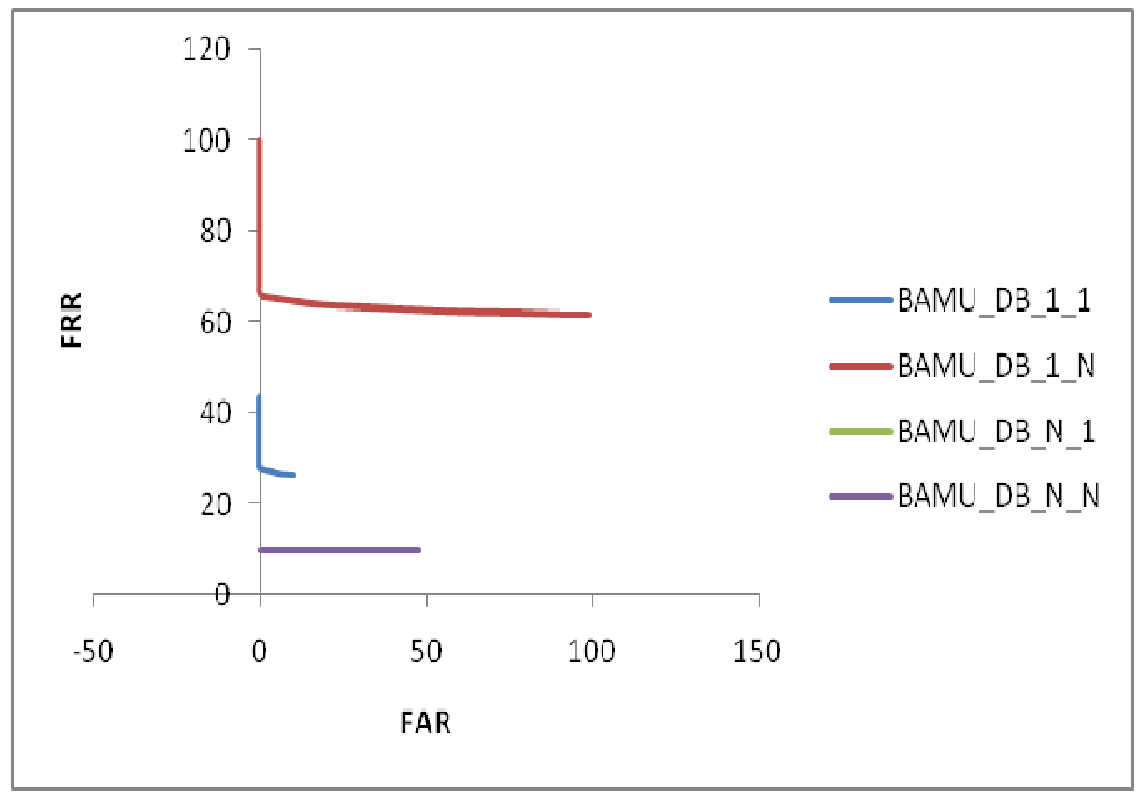

Fig.9: ROC FVC 2004_DB1

The Performance of the fingerprint verification system is measure using FAR and FRR. ROC plot is a visual charactization of the exchange between the FAR and the FRR. In general, the matching algorithm performs a decision based on a threshold which determines how close to a template. If the threshold is reduced, there will be less false non-matches but more false accepts. Similarly, a higher threshold will reduce the FAR but increase the FRR. We also calculate EER which is rate at which both accept and reject errors are equal. The value of the EER can be easily obtained from the ROC curve. Above results show that when we use single one fingerprint for enrollment and one single fingerprint impression for testing (unimodal) results are less as compare with one to multiple, multiple to one and multiple to multiple fingerprint matching. Results with database FVC2000_DB1 we can see that RR is $75 \%$ at one to one matching but it increased by $1.5 \%, 8 \%$ and $5 \%$ in one to multiple, multiple to one and multiple to multiple fingerprint matching respectively, it is found some different in database FVC2002_DB1 where RR is $80 \%$ at one to one matching, it is increased by $8 \%$ in multiple toone matching and no change in all other. Database FVC2004_DB1 results show that RR is 
increase multiple to one matching and multiple to multiple matching which are increase by $1 \%$ and 8.5\%.This is very clear in BAMU_DB RR is 89.5 in unimodal one to one matching but it is increase multiple to one and multiple to multiple matching by $10 \%$ and $4 \%$ respectively.

\section{CONCLUSION}

We have compared the performance of fusion of multiple impressions at different level. We apply Filterbank-base

\section{ACKNOWLEDGMENTS}

Others wish to acknowledge the UGC who have funded for development of UGC-MRP-F.No-33-63/2007(SR), entitle "Development of Multimodal Fingerprint Recognition System Through Multiple Impressions and Matching Technique".

\section{REFERENCES}

[1] Chunyu Yanga and Jie Zhou "A comparative study of combining multiple enrolled samples for fingerprint verification",Elsevier, Pattern Recognition 39 (2006) 2115 -2130 .

[2] A. K. Jain, S. Pankanti, S. Prabhakar, L. Hong, A. Ross, and J. L. Wayman, "Biometrics: A Grand Challenge", Proc. International Conference on Pattern Recognition (ICPR),IEEE, vol. II, pp. 935-942, Cambridge, UK, Aug. 2004.

[3] D. Maltoni, D. Maio, A.K. Jain, and S. Prabhakar, Handbook of fingerprint recognition, New York: Springer, 2003.

[4] Anil K. Jain, Salil Prabhakar, Lin Hong, and Sharath Pankanti," Filterbank-Based Fingerprint Matching", IEEE Transactions on Image Processing, vol. 9, No. 5,May 2000.

[5] Anil K. Jain, Salil Prabhakar," Decision-level Fusion in Fingerprint Verification", Elsevier, Pattern Recognition 35 (2002) 861-874.

[6] Lifeng Sha, Feng Zhao, Xiaoou Tang, A Two-Stage Fusion Scheme using Multiple Fingerprint Impressions, Proceedings of the International Conference on Image Processing, ICIP 2007, (SanAntonio, Texas, USA), September 2007.

[7] Chunxiao Ren, Yilong Yin, Jun Ma, and Gongping Yang,"A Novel Method of Score Level Fusion Using fingerprint algorithm and Score level fusion technique for multiple impressions of same finger. By study on the overall correspondence outputs, we observed that Preliminary experimental results show the less RR with unimodal one to one matching but we observed fusion of multiple impressions of same finger at enrollment or testing level increase the system performance. Hence, in this research work, experiments are conducted on the multimodal biometrics data for convenience.

Multiple Impressions for Fingerprint Verification" Proceedings of the 2009 IEEE International Conference on Systems, Man, and Cybernetics San Antonio, TX, USA October 2009.

[8] Anil K. Jain, Salil Prabhakar, Lin Hong, and Sharath Pankanti," Filterbank-Based Fingerprint Matching", IEEE Transactions on Image Processing, vol. 9, No. 5,May 2000.

[9] Anil K. Jain, Patrick Flynn, Arun A. Ross,'Handbook of Biometrics" Springer,c2008.

[10] Arun Ross," An Introduction to Multibiometrics",appeared in proc.of the 15th European Signal Processing Conference(EUSIPCO), September 2007.

[11] Arun A. Ross, Anil K Jain, Karthik Nandakumar,"Handbook of Multibiometrics" Springer, 2006.

[12] D. Maio, D. Maltoni, R. Cappelli, J. L. Wayman, A. K. Jain,"FVC2000: Fingerprint Verification Competition" IEEE Transactions on Pattern Analysis Machine Intelligence, vol.24, no.3, pp.402-412, March 2002.

[13] D. Maio, D. Maltoni, R. Cappelli, J.L.Wayman, A.K. Jain," FVC2002: Second Fingerprint Verification Competition" in proceedings 16th International Conference on Pattern Recognition (ICPR2002), Québec City, vol.3, pp.811-814, August 2002.

[14] D. Maio, D. Maltoni, R. Cappelli, J.L. Wayman, A.K. Jain," FVC2004: Third Fingerprint Verification Competition" in proceedings International Conference on Biometric Authentication (ICBA04), Hong Kong, pp.1-7, July 2004.

[15] Vikas T. Humbe,'Development of Enhancement Technique for Fingerprint Recognition System" Ph.D. Thesis

2007 